Meta

Journal des traducteurs

Translators' Journal

\title{
Fonctions et structure du langage juridique
}

\section{Georges A. Legault}

Volume 24, numéro 1, mars 1979

La traduction juridique

URI : https://id.erudit.org/iderudit/003456ar

DOI : https://doi.org/10.7202/003456ar

Aller au sommaire du numéro

Éditeur(s)

Les Presses de l'Université de Montréal

ISSN

0026-0452 (imprimé)

1492-1421 (numérique)

Découvrir la revue

Citer cet article

Legault, G. A. (1979). Fonctions et structure du langage juridique. Meta, 24(1),

18-25. https://doi.org/10.7202/003456ar d'utilisation que vous pouvez consulter en ligne.

https://apropos.erudit.org/fr/usagers/politique-dutilisation/ 


\section{Fonctions et structure du langage juridique}

Vers 1700 avant Jésus-Christ, Hammourabi, roi de Babylone, faisait inscrire sur une stèle diorite les lois de son pays. De la même façon, vers 1500 avant Jésus-Christ, Moïse présente à son peuple les tables saintes écrites de la main de Dieu. Certes ces exemples, tirés de l'histoire, sont bien différents de nos législations modernes. Les différences qui existent entre les modes de production, les sphères touchées par la législation, les conditions sociales et économiques qui les génèrent, bref, toutes les transformations sociales, font qu'il est difficile de comparer ces législations d'autrefois avec celles d'aujourd'hui. Cependant, on peut affirmer que la forme écrite que prennent ces législations contrairement à la forme verbale de la coutume privilégiée alors, permet un certain rapprochement avec nos systèmes actuels de législation.

Ce changement de forme peut apparaître à première vue sans conséquence ou sans cause significative. Ne s'agit-il pas en fait d'un changement dans la manière de communiquer les lois, dans le but d'éviter les déformations, les ajouts qui sont toujours possibles avec la coutume? Nous ne cherchons pas à déterminer ici les causes véritables de ce changement mais à indiquer seulement à la lumière de cet exemple, la transformation des représentations qu'on se fait du droit. Ces représentations dépendent de la nature de l'acte de communication choisi. La coutume, à cause de son caractère verbal met en présence les personnes impliquées. Le rapport est donc personnel. Les lois et les usages, bien qu'ils proviennent d'un autre, sont transmis par une personne qui les vit. L'auteur des lois est à la fois transcendant et immanent à la transmission. La forme écrite, au contraire marque avant tout la transcendance de la source des lois. Or, la distinction entre la source et la loi permet des représentations contraires. Elle peut mettre en évidence la source, comme dans l'exemple de Moïse, et permettre ainsi de qualifier cette loi de sacrée. Par contre, elle peut dissimuler la source et son caractère arbitraire humain en présentant la loi comme s'imposant d'elle-même. Dans ce cas, l'accent est mis sur la loi. Tel est notre système juridique contemporain où la source prend le visage de la démocratie ou de la volonté générale.

Avec le règne de la loi, de la loi écrite, on voit apparaître tout un univers juridique. Ce dernier s'étend de plus en plus et transpose chacun des rapports humains en terme mesurable. Or, dans cet univers rien n'est laissé au hasard. 
Puisque la source de droit ne peut être interrogée directement pour savoir quel est le sens précis de tel article ou de telle clause, l'art de l'interprétation juridique consistera à cerner le sens de la loi et l'intention supposée du législateur par l'analyse de la forme écrite. Cette caractéristique de l'interprétation juridique nous indique pourquoi les fonctions de rédacteurs et de traducteurs des lois sont si importantes. On ne peut pas se permettre de traduire une loi comme on traduit un autre texte, car la traduction est aussi loi. C'est pourquoi un étudiant en droit apprend dès les premières années de sa formation à saisir le sens d'une loi en comparant la version française et la version anglaise. En privilégiant l'une d'entre elles, il pourra peut-être soutenir avec succès une argumentation nouvelle pour défendre son point de vue. Ainsi, les deux versions d'une loi font autorité et jusqu'à la loi 101 aucune n'avait préséance sur l'autre.

Jusqu'ici nous avons voulu cerner le caractère formel du droit qui se manifeste dans cette forme écrite utilisant une ou des langues, forme qui se présente comme une réalité historique privée de sa source. Certes on ne retrouve pas dans nos droits contemporains une source bien identifiée qui dit le droit mais cela justifie-t-il que le droit soit considéré comme un langage? Si on définit le langage comme tout système de signes aptes à servir de moyen de communication on peut se demander en quel sens le droit est un langage juridique. Le droit n'est pas un système de signes différent de celui de la langue, ainsi il ne peut être considéré comme un langage que dans un sens métaphorique. En effet, l'absence de source lui donne des caractéristiques telles qu'on peut le considérer comme formant un système de signes différent des autres.

On peut s'interroger sur la pertinence de l'expression « langage juridique » telle qu'énoncée mais plus fondamentalement encore, on peut se demander si effectivement toutes les législations, les jugements, les contrats, etc., peuvent se regrouper sous cette unique expression. En utilisant l'expression \&langage juridique » pour qualifier métaphoriquement la forme juridique, ne présupposonsnous point une constante formelle analogue à celle que cherchent les philosophes lorsqu'ils définissent le droit? Tel est le problème central de la réflexion sur la nature même du droit, que cette réflexion se nomme théorie pure du droit, science du droit ou philosophie du droit ${ }^{1}$. L'ensemble des phénomènes visés par l'expression «le droit» possède-t-il une organisation, une structure qui lui soit propre? On songe ici aux comportements des individus vis-à-vis des objets : achat, vente, location ; vis-à-vis des personnes : mariage, fliation, ou vis-à-vis des institutions judiciaires, législatives ou tout ce que ces institutions regroupent. Or, tous ces phénomènes juridiques peuvent prendre une forme écrite, que ce soit sous forme d'actes privés (contrats) ou d'actes publics (testaments, lois, jugements).

1. Les expressions \& philosophie > et ici \& philosophie du droit " sont souvent investies d'une connotation péjorative. La réaction en science humaine suit celle en science expérimentale où l'on utilise l'expression \& métaphysique » pour qualifier toute recherche qui utilise une méthode différente de la leur, fondée sur autre chose que la vérification expérimentale. L'expression * philosophie du droit \$a donc été malheureusement identifiée à la position métaphysique de droit naturel. 
Pour certains, la recherche d'un principe d'organisation ou d'unification $\mathrm{du}$ droit est illusoire. La multiplicité des lois, par exemple, reflète la diversité des objets sur lesquels on légifère. Est-ce que chaque loi ne possède pas sa terminologie, ses concepts et une structure déterminée par l'objet de législation? Ainsi, comment comparer le Code civil de la province de Québec avec le Code pénal et l'Acte de l'Amérique du Nord britannique ? Cependant, dans l'histoire de la philosophie du droit, les auteurs tels que H. Kelsen, Alf Ross et autres ont essayé d'unifier l'ensemble de ces manifestations juridiques en mettant en évidence les fonctions précises des institutions juridiques et leur étroite interdépendance $^{2}$. D'un certain point de vue chaque acte législatif peut être considéré comme un tout, présentant son propre principe d'organisation en fonction de l'objet de législation. Cependant, il ne faut pas oublier que chaque législation participe à l'ensemble de l'activité législative qui s'adresse aux citoyens et que chaque législation est susceptible d'interprétation judiciaire. Le langage juridique du citoyen et celui des juges sont donc en étroite relation avec celui de la législation, chacun représentant une fonction du droit.

Rechercher l'unification des langages juridiques, nous l'avons souligné, c'est rẹchercher l'unité du droit. Ces deux recherches sont inséparables. On ne peut pas séparer le droit de son expression, c'est pourquoi l'étude du droit par le biais de son langage nous conduit à une interprétation de l'ensemble des phénomènes juridiques. Dans les sections qui vont suivre nous essaierons de préciser brièvement les différentes fonctions attribuées au langage juridique, de voir comment se structure le langage juridique et enfin de mesurer comment la structure performative permet l'unification du droit.

\section{Fonctions du langage juridique}

Le langage juridique peut être étudié de différents points de vue. Certains auteurs l'étudient du point de vue sémantique. D'autres cherchent en définitive une réponse à l'interrogation «Que dit le droit? \$ Ce type d'interrogation dépasse le cadre de l'analyse sémantique car il essaie d'en élucider la fonction pragmatique. En privilégiant l'aspect fonction du langage juridique, ces auteurs doivent, d'une part, unifier les différentes propositions juridiques qu'on retrouve dans les multiples lois et, d'autre part, élaborer un métalangage juridique qui explique la fonction du langage juridique tel qu'on le retrouve dans nos législations. Par exemple, on pourrait prétendre que le langage juridique a une fonction descriptive. Cependant, cette fonction n'est pas celle qu'on retrouve directement dans nos législations. Il suffit de lire quelques articles du Code pénal pour s'en rendre compte. Par contre, certains articles du Code civil utilisent la notion de droit, tel l'article 406 qui définit le droit de propriété.

Ainsi une théorie qui veut démontrer que le langage juridique est descriptif doit donc expliquer comment les dispositions du Code pénal, des codes de procédures et des autres statuts, décrivent des droits. $\bar{A}$ cet égard on pourrait soutenir que les infractions du Code pénal visant les biens ne sont que la pro-

2. Le lecteur pourrait consulter sur ce point la première partie de mon livre, la Structure performative du langage juridique, Montréal, Presses de l'Université de Montréal, 1977. 
tection du droit de propriété, de même que les infractions contre l'intégrité physique d'une personne protègent le droit de la personne. Sans élaborer davantage ce que pourrait être cette théorie, on peut déjà constater une difficulté fondamentale. Si le droit décrit des droits, il faut donc admettre que ces droits des individus sont antérieurs à la législation, qu'ils sont dans notre nature humaine. Or, comment arrivons-nous à la connaissance de ces droits, de ce droit naturel ?

Les philosophies positivistes du droit ont critiqué les théories du droit naturel notamment sur la connaissance possible d'un droit antérieur à la législation. De plus, ces philosophies ont montré que le droit ne fait pas que décrire, mais qu'il crée quelque chose. Par exemple, lorsque le tribunal se prononce sur un litige, il ne dit pas ce que dit le droit. L'interprétation judiciaire et l'application à une situation concrète compte tenu des témoins, des procédures et de la preuve font que le tribunal crée le droit. Mais comment ? Pour Kelsen, le tribunal crée des normes. Ainsi, le langage juridique est un langage normatif.

Selon Kelsen, le langage juridique atteint son but en établissant des normes. En effet, pour diriger la conduite des individus, le droit émet des règles qui doivent être suivies. Mais ces règles ne s'adressent pas premièrement aux individus. Le droit ne dit pas à un individu ce qu'il doit faire ou ce qu'il doit s'abstenir de faire. Le droit pose des normes qui s'adressent premièrement aux juges. La norme prend alors la forme suivante : Si tel acte est illicite alors telle sanction doit être. Puisque le juge applique cette norme à des situations concrètes en déclarant que telle sanction doit être exécutée (emprisonnement, saisie), les individus vivent cette norme comme une interdiction \& Tu ne dois pas », comme un đevoir \& Tu dois » ou comme un droit lorsqu'on ne prévoit pas de sanction à un acte, «Tu peux ».

Ces deux exemples, quoique bien peu développés, nous montrent que l'analyse du langage juridique est très complexe car les fonctions sont multiples. D'une part, le langage s'adresse aux individus, à tous ceux qui sont sur le territoire et il s'adresse aussi aux juges. D'autre part, il semble définir des mots et des droits, déclarer des droits, permettre et interdire des conduites. C'est pourquoi les théories descriptives et normatives du langage juridique semblent réduire le droit à une seule dimension.

\section{Structure et fonctions du langage juridique}

On ne peut répondre à la question \&Que dit le droit? \& sans d'abord déterminer ce que « dire » veut dire. Les premiers philosophes qui se sont intéressés à ce sujet ont considéré que la seule fonction significative du langage était celle de la description. Or, il a fallu un J.L. Austin pour ouvrir le champ d'interrogation sur le langage à autre chose que le jugement de fait et le jugement de valeur. Austin jugeait, avec raison, que ces deux catégories d'énoncés ne pouvaient tout expliquer et particulièrement les énoncés comme ceux de la promesse. En effet, lorsque je dis que je promets de faire quelque chose, je ne décris pas ni n'évalue cette chose. Par contre, par cet énoncé, je crée quelque 
chose, je m'engage à faire une action quelconque dans l'avenir. Pour distinguer ce genre d'énoncés des autres, Austin les nomme des «performatifs».

Dans son livre How to Do Things with Words ${ }^{3}$, Austin développe dans une première partie la comparaison entre constatifs (énoncés descriptifs) et performatifs. Cependant, cette comparaison lui ouvre une nouvelle voie. Alors que les performatifs semblent être une catégorie d'énoncés différente des constatifs, l'analyse des performatifs le conduit à reconnaître que tous les énoncés peuvent être considérés comme des performatifs. Généralisant les caractéristiques d'une catégorie d'énoncés à tous les énoncés, il modifie en conséquence sa terminologie et il élabore ainsi une théorie des forces du discours. Poursuivant l'analyse d'Austin, J. Searle développe une théorie des Speech Acts ${ }^{4}$, des actes de parole ou plus précisément des actes de discours.

Selon cette théorie des actes de discours, « dire » apparaît comme un comportement dirigé par certaines règles. En plus des règles sémantiques, linguistiques, syntaxiques, parler obéit à des règles qui déterminent l'acte de discours utilisé. Chaque acte de discours est donc susceptible de réussite ou d'échec selon que les règles en question sont respectées ou non. Certes, les auteurs ne sont pas unanimes sur les règles particulières de chaque acte de discours ${ }^{5}$. Cependant, on peut au moins préciser certaines constantes assez générales. Chaque acte de discours suit un certain nombre de règles. Selon les auteurs ces règles sont plus ou moins nombreuses ou bien elles sont décrites différemment. Cependant, on reconnaît au moins l'importance des règles suivantes : la règle fondamentale et les règles préparatoires. La règle fondamentale est conventionnelle et elle précise la fonction de l'acte de discours. Par exemple, la règle fondamentale de la promesse précise que l'énoncé met le locuteur dans l'obligation d'effectuer l'acte promis. Les règles préparatoires viennent préciser certaines conditions nécessaires à la réussite de l'acte. Ces règles regroupent selon moi, deux séries de conditions, celles relatives à la capacité d'émettre l'énoncé et celles relatives à l'objet même de l'énoncé. Pour ordonner à quelqu'un de faire quelque chose, par exemple, il faut que la personne qui émet l'énoncé soit en position d'autorité. Ceci est un exemple de règle de capacité. De même, je ne peux ni ordonner, ni promettre n'importe quoi, il faut que l'action soit un acte futur. C'est là une règle d'objet. On comprend facilement que l'acte ne peut pas réussir sans le respect de ces règles.

Ainsi, « dire » c'est faire des actes en suivant certaines règles. Mais comment ces constatations sur le dire permettent-elles de répondre à la question initiale «Que dit le droit? 》 Peut-on effectivement interpréter l'ensemble du langage juridique d'après le modèle de la description ou du commandement comme l'ont tenté les théories mentionnées? Ce que l'analyse du langage quotidien présente permet davantage. Dire n'est pas un seul acte mais une pluralité

3. J.L. Austin, How to Do Things with Words, Londres, Oxford University Press, 1962.

4. J. Searle, Speech Acts. An Essay in the Philosophy of Language, New York, Cambridge

University Press, 1970.

5. La deuxième partie de mon livre présente une analyse critique des conceptions d'Austin et de Searle. Cette critique permet l'élaboration d'un modèle d'acte de discours compatible avec le langage juridique. C'est ce modèle que j'ai nommé la structure performative. 
d'actes. Pourquoi le langage juridique serait-il différent? Pourquoi essayer d'interpréter ce langage d'après un seul acte de discours ? Par contre, si on reconnaît au droit cette pluralité d'actes analogues à ceux du langage, ne consacre-ton pas la multiplicité du droit et n'avoue-t-on pas l'impossibilité d'en dégager une unité ?

Ce qui permet l'unité du droit malgré la diversité des actes c'est leur structure performative. Les actes du langage juridique ressemblent aux actes de discours présentés. Comme eux, ils se composent d'une règle fondamentale, des règles de capacité et d'objet. Mais ces règles ne sont pas suffisantes, ni pour les actes de discours ni pour les actes juridiques. Il faut aussi des règles relatives à la communication des actes, car des erreurs dans la communication, émission ou réception, conduisent inévitablement à un échec de l'acte en question. Cependant, ce qui distingue les actes juridiques des actes de discours, c'est la règle fondamentale ${ }^{B}$. En effet, cette dernière précise toujours que l'accomplissement de certains gestes, crée quelque chose. Les actes juridiques sont créateurs de faits, de droits et d'obligations. C'est pourquoi le mot «performatif » d'Austin est utilisé dans l'expression «structure performative du langage juridique ».

\section{Droit et structure performative du langage juridique}

D'un certain point de vue, le droit apparaît essentiellement comme un phénomène législatif, où les lois déterminent la conduite des individus. D'un autre point de vue, le droit apparaît essentiellement comme un phénomène judiciaire. Le droit c'est l'application réelle, jugement, prison, saisie, etc. C'est pourquoi, la législation serait un ensemble de règles permettant un jugement déterminé. Or, comment concilier ces trois aspects du droit : législation, comportement des citoyens, activité judiciaire?

La structure des actes de discours nous a permis de voir que \& dire » est un comportement régi par des règles. De manière analogue, le droit est constitué d'actes, qui sont réalisés par des individus. Puisque certaines personnes ont une fonction bien précise dans le système juridique, tels les députés, les juges, ces personnes sont soumises à la fois aux règles générales applicables à tout citoyen et à celles relatives à leur fonction. La fonction législative se résume donc à élaborer des structures performatives qui, grâce à la règle fondamentale, créent un fait, une ou des obligations ou un droit ?

$\mathrm{Si}$ on regarde la Constitution, on constate qu'elle pose les règles déterminant les actes de législation et les actes judiciaires. Ainsi les lois qui seront présentées mais qui n'auront pas respecté la règle fondamentale et les autres règles de cet acte de législation seront annulables par les tribunaux. De même, les lois poseront à leur tour des structures performatives pour les individus. Lorsqu'on

6. La règle fondamentale des actes juridiques se distingue de celle des actes de discours telle que celle-ci est interprétée par Austin et Searle. Personnellement, je crois possible l'interprétation de tout acte de discours selon le modèle performatif. Cette hypothèse, bien qu'appuyée par des exemples dans mon livre, n'est cependant pas suffisamment élaborée pour prouver une conclusion ferme sur ce point.

7. La troisieme partie de mon livre est consacré à l'exposition des règles des principaux actes juridiques. 
regarde au niveau du droit civil, le contrat, le quasi-contrat, les testaments, le mariage, on voit que les articles du Code précisent dans chacun des cas quels gestes, signes ou faits produits par des individus créent un fait, un droit ou une obligation. Dans la cérémonie du mariage, l'échange des consentements crée le fait d'être marié, ce qui change mon statut. En plus de ce fait, l'échange des consentements crée une série d'obligations. Ainsi, les articles du Code civil précisent les règles de capacité et d'objet, la règle fondamentale et les règles de communication pour que les actes juridiques soient réussis. C'est pourquoi la personne qui désire la création de cette obligation ou de ce nouveau fait, devra respecter les règles de l'acte qui le crée.

Dans cet univers juridique, beaucoup de nos obligations sont respectées et s'éteignent par l'accomplissement réciproque des prestations. Cependant, dans certains cas, l'obligation n'est pas respectée. Par exemple, un acheteur ne paie pas ses mensualités. Or, plusieurs raisons peuvent expliquer ce fait. D'une part l'acheteur peut refuser de payer parce que l'obligation du cocontractant n'est pas remplie, d'autre part l'acheteur peut contester la validité du contrat et l'existence même de son obligation. Dans ces cas litigieux on s'adresse aux tribunaux. Pour ce faire, les parties devront suivre un ensemble de règles qui déterminent " la contestation liée " qui seule est susceptible de jugement. Ainsi, les codes de procédures précisent d'autres structures performatives. Le tribunal ayant suivi les règles de la preuve, détermine si les règles de la structure performative de l'acte juridique, dans notre exemple, le contrat, ont effectivement été respectées. Si le tribunal déclare l'acte réussi, il exige que l'obligation soit accomplie. $\mathrm{Si}$, malgré ce jugement la personne ne respecte pas son obligation, alors on peut demander l'exécution forcée du jugement rendu.

En droit pénal, la situation est différente. Les structures performatives présentées visent des comportements que la société bannit. Bien qu'on y retrouve la structure performative comme dans les actes juridiques de droit civil, la règle fondamentale des performatifs pénaux se distingue de ceux de droit civil parce que son effet n'est pas immédiat. Si l'accomplissement de tel ou tel geste nous rend «coupable de meurtre» par exemple, ce fait, la culpabilité légale, n'est pas immédiatement réalisé. La présomption d'innocence intervient en droit pénal de manière à rendre la culpabilité légale conditionnelle au jugement. C'est le tribunal qui déclare l'individu coupable et de ce fait crée par le performatif du jugement cette culpabilité légale. Comme en témoigne le procès par jury au niveau criminel, la détermination de la culpabilité relève du jury alors que la détermination de la sanction revient au juge.

En droit civil comme en droit pénal, le jugement est aussi créateur de droit. Tout jugement suit des règles qui déterminent qui a la capacité de juger et quel objet peut être jugé par le tribunal. De plus, le procès se déroule conformément aux règles relatives à l'audition de la cause. Or, le jugement crée des droits, des obligations ou des faits. Comme tout autre acte juridique, une des règles peut ne pas avoir été respectée, c'est pourquoi il existe des tribunaux d'appel. Puisqu'on peut régresser à l'infini, de tribunal d'appel à un autre tribunal d'appel, 
on stipule par législation que tel tribunal rendra des déclarations finales, telle est la fonction par exemple de la Cour suprême du Canada.

«Que dit le droit? \$ Le droit ne peut être réduit à un ensemble de droit ou de normes. Beaucoup plus riche que cela, le droit est comme notre langage, constitué d'un ensemble d'actes. En effet, du point de vue du métalangage le droit présente les règles qu'il faut suivre pour obtenir tel résultat conventionnel souhaité ou les comportements à éviter si on ne désire pas tel résultat conventionnel. Non seulement chaque acte est défini par des règles mais encore chacun d'eux est relié aux autres. C'est pourquoi la structure des institutions juridiques paraît pyramidale avec la constitution pour sommet et les comportements des individus pour base.

Cette conception du droit décrit seulement un des aspects du droit, la forme juridique. Cette forme prit l'aspect d'un langage sans source avec la forme écrite et aussi la forme magique avec le droit romain. La dimension formelle du droit est certes un aspect important ; cependant, après l'avoir décrit on se demande comment et pourquoi les hommes ont créé cette forme pour régir leurs comportements.

Georges A. Legault 